\title{
Comparison of optimised endovaginal vs external array coil T2-weighted and diffusion-weighted imaging techniques for detecting suspected early stage (IA/IB1) uterine cervical cancer
}

\author{
Kate Downey $^{1}$ • Ayoma D. Attygalle ${ }^{2}$ - Veronica A. Morgan ${ }^{1} \cdot$ Sharon L. Giles ${ }^{1}$. \\ A. MacDonald ${ }^{1}$ • M. Davis ${ }^{4}$ - Thomas E. J. Ind ${ }^{3} \cdot$ John H. Shepherd $^{3}$. \\ Nandita M. deSouza ${ }^{1}$
}

Received: 24 March 2015 / Revised: 9 June 2015 / Accepted: 22 June 2015 /Published online: 11 July 2015

(C) The Author(s) 2015. This article is published with open access at Springerlink.com

\begin{abstract}
Objective To compare sensitivity and specificity of endovaginal versus external-array coil T2-W and T2-W+ DWI for detecting and staging small cervical tumours.

Methods Optimised endovaginal and external array coil MRI at 3.0-T was done prospectively in 48 consecutive patients with stage $\mathrm{Ia} / \mathrm{Ib} 1$ cervical cancer. Sensitivity/specificity for detecting tumour and parametrial extension against histopathology for a reading radiologist were determined on coronal T2-W and T2W+DW images. An independent radiologist also scored T2-W images without and with addition of DWI for the external-array and endovaginal coils on separate occasions $>2$ weeks apart. Cohen's kappa assessed inter- and intraobserver agreement.

Results Median tumour volume in 19/38 cases positive on subsequent histology was $1.75 \mathrm{~cm}^{3}$. Sensitivity, specificity, PPV, NPV were: reading radiologist $91.3 \%, 89.5 \%$, $91.3 \%, 89.5 \%$, respectively; independent radiologist T2-W $82.6 \%, 73.7 \%, 79.1 \%, 77.8 \%$ for endovaginal, $73.9 \%$, $89.5 \%, 89.5 \%, 73.9 \%$ for external-array coil. Adding DWI
\end{abstract}

Nandita M. deSouza

nandita.desouza@icr.ac.uk

1 CRUK Cancer Imaging Centre, The Institute of Cancer Research and Royal Marsden NHS Foundation Trust, Downs Road, Surrey SM2 5PT, UK

2 Department of Histopathology, The Royal Marsden NHS Foundation Trust, Fulham Road, London SW3 6JJ, UK

3 Gynecology Unit, The Royal Marsden NHS Foundation Trust, Fulham Road, London SW3 6JJ, UK

4 Department of Gynaecology, Kingston Hospital, Galsworthy Road, Kingston-upon-Thames, Surrey KT2 7QB, UK improved sensitivity and specificity of endovaginal imaging (78.2 \%, 89.5\%); adding DWI to external-array imaging improved specificity $(94.7 \%)$ but reduced sensitivity $(66.7 \%)$. Inter- and intra-observer agreement on T2-W+DWI was good (kappa $=0.67$ and 0.62 , respectively).

Conclusion Endovaginal coil T2-W MRI is more sensitive than external-array coil for detecting tumours $<2 \mathrm{~cm}^{3}$; adding DWI improves specificity of endovaginal imaging but reduces sensitivity of external-array imaging.

Key Points

- Endovaginal more accurate than external-array T2-W MRI for detecting small cervical cancers.

- Addition of DWI improves sensitivity and specificity of endovaginal T2-W imaging.

- Addition of DWI substantially reduces sensitivity of externalarray $T 2-W$ imaging.

Keywords Cervical cancer · Endovaginal coil · External array coil · Diffusion-weighted MRI · Fertility

$\begin{array}{ll}\text { Abbreviations } \\ \text { ADC } & \text { Apparent diffusion coefficient } \\ \text { DWI } & \text { Diffusion-weighted imaging } \\ \text { EPI } & \text { Echo-planar imaging } \\ \text { FIGO } & \text { Federation Internationale Gynecology and } \\ & \text { Obstetrics } \\ \text { FSE } & \text { Fast spin-echo } \\ \text { LLETZ } & \text { Large loop excision of the transformation zone } \\ \text { MRI } & \text { Magnetic resonance imaging } \\ \text { SENSE } & \text { Sensory encoding } \\ \text { SNR } & \text { Signal-to-noise ratio } \\ \text { TSE } & \text { Turbo spin-echo }\end{array}$


ZOOM Zonal oblique multislice

PPV Positive predictive value

NPV Negative predictive value

RVT Radical vaginal trachelectomy

\section{Introduction}

Increasingly, because of effective cytological screening programmes, uterine cervical cancer presents at an early stage with small-volume disease. A 'see-and-treat' policy at most centres results in referrals for imaging following a diagnostic cone biopsy or large loop excision of the transformation zone (LLETZ) procedure, at which a significant proportion of the disease may be excised. The presence of residual disease is critical to inform further surgical management especially if considering preservation of fertility, so that its identification has become a key requirement from imaging. Cervical amputation (trachelectomy) is an option in these cases, where fertility is not compromised for other reasons [1] but requires at least a $1-\mathrm{cm}$ length of normal cervical canal between the superior tumour margin and the internal os $[2,3]$. Therefore, knowledge of residual tumour margins in relation to the normal cervical anatomy $[4,5]$ is paramount when considering patients for trachelectomy. The superior soft-tissue contrast provided by magnetic resonance imaging (MRI) makes it the imaging technique of choice for demonstrating cervical tumour in relation to normal cervical anatomy [6] in preoperative imaging [7]; standard image acquisition using external phased array receiver coils at field strengths of 1.5-T or 3.0T results in an in-plane resolution of $>1 \mathrm{~mm}$ with a 3- to 4-mm slice thickness. At this resolution, tumours of $>1 \mathrm{~cm}$ have a sensitivity and sensitivity of detection around $60 \%$, although specificity is high $[8,9]$, but smaller tumours can be missed because spatial resolution is limited by the signal-to-noise ratio (SNR) available.

The spatial resolution of MR images may be improved by the use of receiver coils close to the region of interest because they provide significant increases in SNR [10-12]. The use of an endovaginal receiver coil has previously been shown to improve the detection of tumours of $<1.0 \mathrm{~cm}^{3}$ on T2-W imaging [13] and also allows detailed visualisation of the parametrium up to $6 \mathrm{~cm}$ from the surface of the internal coil [14]. When combined with diffusion-weighted (DW) contrast, the endovaginal technique further improves sensitivity and specificity of local staging to $92 \%$ and $82 \%$, respectively $[15,16]$ because the apparent diffusion coefficient (ADC) of tumour is significantly lower than normal tissue [17]. However, a formal comparison of an optimised $\mathrm{T} 2+\mathrm{DW}$ protocol for tumour detection using an endovaginal versus an external array-only technique has hitherto not been undertaken because of the time and resource implications of doing both fully optimised protocols in the same patients prior to surgery.
The purpose of this work therefore was to undertake such a prospective study and compare the sensitivity and specificity of T2-W and T2-W+DWI obtained using an endovaginal coil with that using an external array coil for detecting smallvolume cervical tumours and identifying early parametrial extension.

\section{Methods}

\section{Endovaginal coil}

A 37-mm ring-design solenoidal receiver coil similar to one previously described for use at 1.5-T [18] was used as part of a coil array with an externally placed flex-L coil (Philips Medical Systems, Best, The Netherlands). The endovaginal coil was designed and built in-house. It was covered in a disposable sheath and required simple manual insertion, without visual or imaging guidance, and manipulated so that the ring encompassed the cervix. The coil was immobilised in an external clamp as previously described. Vaginal air introduced during insertion was aspirated using a Ryles tube to minimise image distortion.

\section{External array}

A receive-only SENSE XL Torso coil 16-element phased array was used (Philips Medical Systems). This is a flexible volume coil consisting of anterior and posterior sections, each of eight elements - four upper and four lower. Coil dimensions were $74 \mathrm{~mm} \times 558 \mathrm{~mm} \times 600 \mathrm{~mm}$ for each half (upper and lower). Each element had a $\mathrm{Z}$ coverage of $22.5 \mathrm{~cm}$.

\section{Sequence optimisation}

We initially established the most appropriate imaging protocols for both the endovaginal technique and the external array technique. As high a resolution as possible with the T2-W sequences without compromising image quality was deemed to be a minimum requirement. We matched the voxel size of the external array imaging to the endovaginal DW imaging (Table 1). From our experience in reporting these images, planes sagittal and coronal to the cervix were selected. The endovaginal images had a spatial resolution of $0.35 \mathrm{~mm}^{3}$ $(0.42 \times 0.42 \times 2 \mathrm{~mm})$, while the torso array had a spatial resolution of $3 \mathrm{~mm}^{3}$ acquired $(1 \times 1 \times 3 \mathrm{~mm})$. In order to minimise acquisition time, DWI for comparison was done in the coronal plane only as parametrial extension is best demonstrated in this plane. For the endovaginal technique we used the ZOOM sequence [19] which substantially reduces image distortions that arise due to susceptibility artefacts from the endovaginal coil. This allows a spatial resolution on the DW images of $3.1 \mathrm{~mm}^{3}(1.25 \times 1.25 \times 2 \mathrm{~mm})$. Because of the lower image 
Table 1 Sequence parameters used for imaging

\begin{tabular}{|c|c|c|c|c|}
\hline Parameter & T2-weighted coronal, sagittal & DWI coronal & T2-weighted & DWI \\
\hline Coil selection & $\begin{array}{l}\text { Dual: Endovaginal+FLEX-L } 2 \text { element } \\
\text { phased array body }\end{array}$ & Single: Endovaginal & SENSE-XL torso & SENSE-XL torso \\
\hline Sequence & $\begin{array}{l}\text { Turbo Spin Echo-Multi Shot } \\
\text { (TSE factor 10) }\end{array}$ & $\begin{array}{l}\text { Spin-Echo Echo Planar } \\
\text { Imaging-Single Shot }\end{array}$ & TSE (TSE Factor 13) & EPI \\
\hline $\mathrm{TR}(\mathrm{ms})$ & $\begin{array}{l}2750 \text { (coronal) } \\
2500 \text { (sagittal) }\end{array}$ & 6500 & 2100 & 3230 \\
\hline $\mathrm{TE}(\mathrm{ms})$ & 80 & 54 & 90 & 53 \\
\hline Flip angle & $90^{\circ}$ & $90^{\circ}$ & $90^{\circ}$ & $90^{\circ}$ \\
\hline Bandwidth (Hz/Px) & 217.7 & 9.6 & 217.3 & 9.6 \\
\hline FOV (mm) & $100 \times 100$ & $100 \times 100$ & $120 \times 200$ & $110 \times 100$ \\
\hline Acq pixel size $(\mathrm{mm})$ & $0.42 \times 0.42$ & $1.25 \times 1.25$ & $1.0 \times 1.0$ & $1.25 \times 1.25$ \\
\hline Acq matrix & 238 & 80 & 120 & 88 \\
\hline Recon matrix & 288 & 224 & 400 & 224 \\
\hline Acq voxel size $\left(\mathrm{mm}^{3}\right)$ & $0.35[0.42 \times 0.42 \times 2]$ & $3.125[1.25 \times 1.25 \times 2]$ & $3.00[1 \times 1 \times 3]$ & $4.69[1.25 \times 1.25 \times 3]$ \\
\hline Slice thickness (mm) & 2 (0.1 separation) & $2(0.1$ separation $)$ & 3 & 3 \\
\hline Recon voxel size $\left(\mathrm{mm}^{3}\right)$ & $0.24[0.35 \times 0.35 \times 2]$ & $0.40[0.45 \times 0.45 \times 2]$ & $0.75[0.5 \times 0.5 \times 3]$ & $0.72[0.49 \times 0.49 \times 3]$ \\
\hline Number of slices & $\begin{array}{l}24 \text { (coronal) } \\
22 \text { (sagittal) }\end{array}$ & 24 & 16 & 16 \\
\hline NSA & 2 & 1 & 4 & 2 \\
\hline $\begin{array}{l}\text { Included b-values } \mathrm{s} / \mathrm{mm}^{2} \\
\text { isotropic }\end{array}$ & $\mathrm{n} / \mathrm{a}$ & $0,100,300,500,800$ & $\mathrm{n} / \mathrm{a}$ & $0,100,300,500,800$ \\
\hline Acquisition time & $\begin{array}{l}4 \min 18 \mathrm{~s} \text { (coronal) } \\
4 \min 0 \mathrm{~s} \text { (sagittal) }\end{array}$ & $4 \min 33 s$ & $3 \min 58 \mathrm{~s}$ & $3 \min 50 \mathrm{~s}$ \\
\hline $\begin{array}{l}\text { Signal in gel }(\mathrm{T} 1=500 \mathrm{~ms}, \\
\mathrm{T} 2=50 \mathrm{~ms}) \text { to background noise }\end{array}$ & $\begin{array}{l}\text { Flex-L elements } 15 \mathrm{~cm} \text { apart, gel sited } \\
\text { centrally within endovaginal coil ring } \\
135\end{array}$ & $\begin{array}{l}\text { Gel sited centrally within } \\
\text { endovaginal coil ring } \\
51\end{array}$ & $\begin{array}{l}\text { Coil elements } 15 \mathrm{~cm} \text { apart, } \\
\text { gel sited centrally } \\
130\end{array}$ & $\begin{array}{l}\text { Coil elements } 15 \mathrm{~cm} \text { apart, } \\
\text { gel sited centrally }\end{array}$ \\
\hline
\end{tabular}

$D W I$ diffusion-weighted imaging, NSA number of signal averages

SNR without the internal coil, it was not possible to derive any meaningful data with the ZOOM-DWI. A conventional echoplanar imaging (EPI) sequence with a pixel resolution equivalent to that of the ZOOM-DWI image was therefore selected $(1.25 \times 1.25 \mathrm{~mm})$ but a slice thickness of $3 \mathrm{~mm}$ was selected to match the slice thickness of the T2-W images and retain SNR, giving a voxel size of $4.7 \mathrm{~mm}^{3}$. Details of the endovaginal coil and external array coil protocols are given in Table 1. SNR measurements were made in a test-object with each coil combination using the optimised sequences and corresponding reconstruction filters, multichannel reconstruction and parallel imaging. A $2.5-\mathrm{cm}$ diameter gel test object (Eurospin, $\mathrm{T} 1=$ $500 \mathrm{~ms}, \mathrm{~T} 2=50 \mathrm{~ms}$ ) surrounded by mineral oil test objects to mimic a body load was imaged with the endovaginal-flex-L coil combination followed by the external array coil, repeating each sequence three times. Mean signal intensity measured from a $200 \mathrm{~mm}^{2}$ region of interest on the central slice within the gel test object from the three repetitions was divided by the mean of the standard deviation of background noise. There was near equivalence of the SNR on T2-W images with each coil combination. There was a $62 \%$ reduction in SNR on the $\mathrm{b}=800 \mathrm{~s} / \mathrm{mm}^{2}$ ZOOM-DWI images from the endovaginal coil compared to the corresponding $\mathrm{T} 2-\mathrm{W}$ images, but a seven times reduction in SNR on the $b=800 \mathrm{~s} / \mathrm{mm}^{2}$ DWI from the external array coil compared to the endovaginal DWI images (Table 1).

\section{Patients}

The study was approved by the local Institutional Review Board. All patients gave informed, written consent. Over a 15-month period (May 2013-Aug 2014), a further followon cohort from our previous study $[15,20]$ of 51 patients were referred for MRI following an abnormal smear test and subsequent histological evidence of invasive cervical cancer. Twenty-two complained of at least one episode of abnormal bleeding unrelated to any preceding surgical procedure. All were successfully prospectively recruited for assessment with endovaginal T2-W MRI followed by high-resolution external phased array MRI at 3.0-T. Three patients were staged as Federation Internationale Gynecology and Obstetrics FIGO 2B at examination under anaesthesia, so that 48 consecutive patients with Stage 1 cervical cancer aged $24-53$ years (mean $34.4 \pm 8.2$ years) were included in this study (Fig. 1). Forty-two patients had a cone or LLETZ biopsy at a median of 4.0 weeks (range 2-12 weeks) prior to MRI in which some or the entire tumour may have been excised; at histology, 26 were 
squamous cell carcinoma and 16 adenocarcinoma. The other five had punch biopsies only (three squamous cell carcinoma, two adenocarcinomas); of these three had undergone cone or LLETZ biopsies more than 9 months previously.

\section{Imaging methods}

Images were obtained at 3.0-T (Philips Achieva). Hyoscine butyl bromide (Buscopan) $20 \mathrm{mg}$ intramuscularly (IM) was administered to reduce artefact from bowel peristalsis.

Endovaginal imaging was performed first. T2-W images were acquired in three orthogonal planes to the cervix and covered it entirely. DWI slice-matched images were acquired in free-breathing coronal to the cervix as parametrial extension is best assessed in this plane. Sequence parameters and acquisition time are given in Table 1. Isotropic apparent diffusion coefficient (ADC) maps based on the monoexponential rate of signal decay with increasing diffusion weighting were generated with the system software (Philips Medical Systems) using all b-values.

Following the endovaginal imaging, the coil was removed and, following a 5-minute off-bed break, external array coil imaging was done. T2-W images were acquired in planes sagittal and coronal to the cervix. DW-slice-matched images were obtained coronal to the cervix. As with the endovaginally acquired data, isotropic ADC maps based on the monoexponential rate of signal decay with increasing diffusion weighting were generated with the system software (Philips Medical Systems) using all b-values.

\section{Data analysis}

At the time of clinical image reading, the reporting radiologist (20 years' experience of pelvic MRI), with access to clinical information and all imaging data sets noted the absence or presence of tumour and the absence or presence of tumour invasion into the right and left parametrium. Where tumour was noted, a tumour volume was measured by drawing a region-of-interest around the tumour on every slice on which it appeared and multiplying the total measured tumour area by the slice thickness plus interslice gap.

On completion of the patient accrual, an independent radiologist (5 years' experience of pelvic MRI) without access to the clinical data separately scored first the anonymised coronal and sagittal T2-W external array images as positive or negative for tumour within the cervix; the absence or presence of parametrial extension was also noted as absent or present. This was then repeated with inclusion of the corresponding coronal ADC maps. At a subsequent occasion 2 weeks later to avoid memorisation, the same observer scored the randomized, anonymised coronal and sagittal T2-W endovaginal images without and then with the addition of the corresponding coronal ADC maps. The absence or presence of right and left parametrial extension was also noted.

\section{Histopathological analysis}

Tissue was acquired at radical hysterectomy $(n=10)$, radical vaginal trachelectomy (RVT) $(n=13)$, cold knife cone $(n=11)$ or LLETZ procedure $(n=4)$ in 38 patients. Of the
Fig. 1 Flow chart of patients included in the study and their clinical pathway. MRI magnetic resonance imaging, $D W-M R I$ diffusion-weighted MRI, FIGO Federation Internationale Gynecology and Obstetrics, LLETZ Large loop excision of the transformation zone

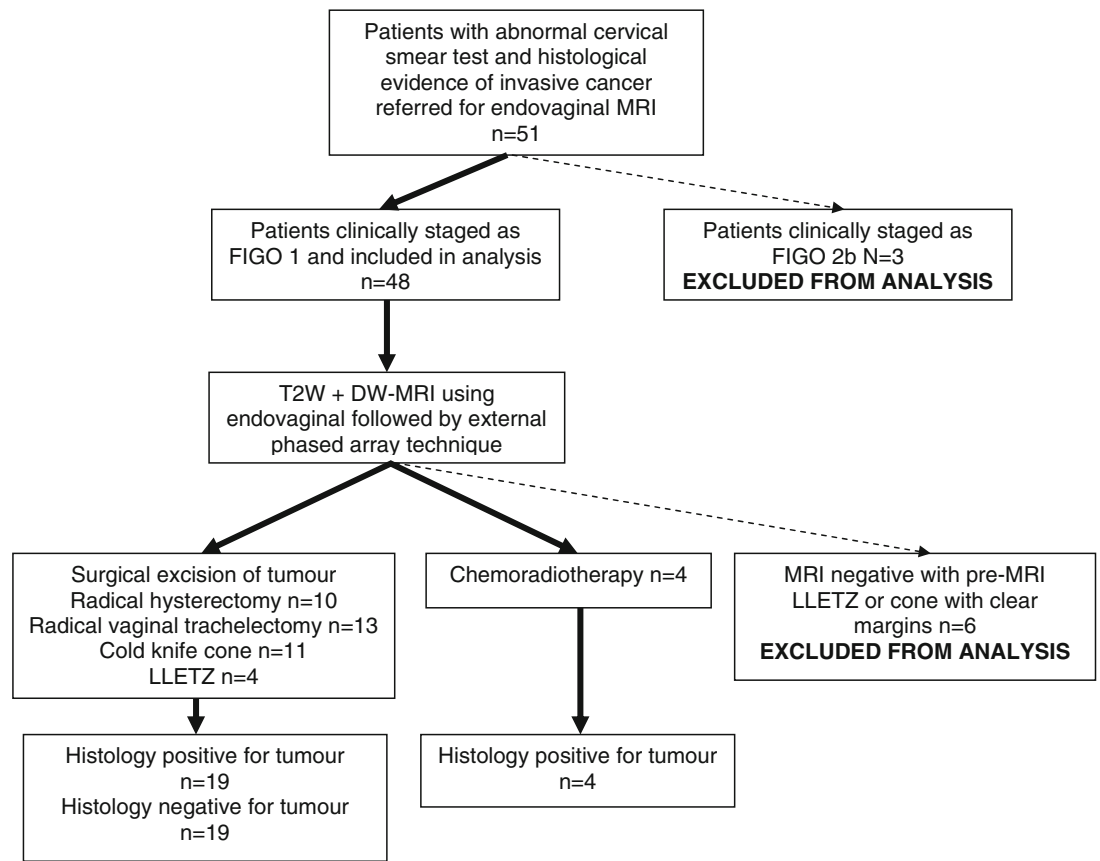


remainder, four went to chemoradiotherapy and were known to have prior positive biopsies and six were deemed to have negative MRI scans with clear margins on the initial cone or LLETZ biopsy so no further intervention at that point was deemed necessary. These latter six cases were therefore excluded from the analysis because of a lack of post-MRI confirmation of the absence of tumour. All specimens were fixed in formalin. For hysterectomy and RVT specimens, a shave of the proximal endocervical resection margin (at or near the internal os) was sliced longitudinally (sagittal plane centrally, coronal plane laterally). The cervix together with the parametrium was sliced into transverse sections up to approximately $1 \mathrm{~cm}$ from the ectocervix. The remaining $1 \mathrm{~cm}$ of distal cervix together with vaginal cuff was sectioned longitudinally (sagittal plane centrally, coronal plane laterally or sagittally throughout). All slices (each 3-4 mm thick) were processed and embedded in paraffin; 2-3 micron sections were deparaffinised and stained with haematoxylin and eosin. According to the thickness of the slice obtained at macroscopy, each histology slide was separated from the next by $3-4 \mathrm{~mm}$.

\section{Statistical analysis}

Sensitivity, specificity, and positive and negative predictive values for detecting the presence of tumour by the reading radiologist were obtained. For the independent radiologist blinded to the clinical information sensitivity, specificity, and positive and negative predictive values of T2-W without and with the addition of DWI were established for detecting the presence of tumour. Data were obtained for the endovaginal technique and separately for the external array-only technique. Similar data were derived for right and left parametrial extension. A Cohen's kappa to assess agreement between observers for interpreting endovaginal $\mathrm{T} 2-\mathrm{W}+\mathrm{DW}$ imaging together was calculated between reading and independent radiologists and between the internal and external coil readings of the independent radiologist on $\mathrm{T} 2-\mathrm{W}$ and separately $\mathrm{T} 2-\mathrm{W}+\mathrm{DW}$ imaging.

\section{Results}

Thirty-eight patients (ten Stage Ia, 28 Stage 1b) underwent a surgical procedure a median of 27 days (lower quartile 23 days, upper quartile 35 days) after MRI; of these, 19 had residual tumour and 19 had no residual tumour on histology. Four patients who received chemoradiotherapy had tumour on biopsy at staging examination under anaesthesia (Fig. 1). Median tumour volume measured on endovaginal MRI of 23 true positive lesions (Fig. 2) was $1.75 \mathrm{~cm}^{3}$ (lower quartile $0.77 \mathrm{~cm}^{3}$, upper quartile $5.5 \mathrm{~cm}^{3}$ ).

Sensitivity and specificity for detecting tumour by the reporting radiologist were $91.3 \%$ and $89.5 \%$, respectively. There were two false positives (Fig. 3) with tumour volumes estimated as $0.05 \mathrm{~cm}^{3}$ and $1.5 \mathrm{~cm}^{3}$, the latter described as a flat ectocervical plaque. The two false negative lesions (Fig. 4) were $6 \mathrm{~mm}$ in maximum dimension on histology. Sensitivity and specificity for detecting tumour by the independent radiologist blinded to the clinical information on endovaginal $\mathrm{T} 2-\mathrm{W}$ alone was $82.6 \%$ and $73.7 \%$; inclusion of the DWI images showed a small reduction in sensitivity to $78.2 \%$ but improved specificity to $89.5 \%$. In comparison, the external array $\mathrm{T} 2-\mathrm{W}$ images showed a lower sensitivity for detecting tumour of $73.9 \%$ but a high specificity of $89.5 \%$. Addition of the DWI images improved specificity further to $94.7 \%$ but at the expense of a substantial loss in sensitivity to $66.7 \%$ (Table 2). The agreement between observers for interpreting endovaginal T2$\mathrm{W}+\mathrm{DWI}$ images together was good (Cohen's kappa= 0.67). Agreement between the independent radiologist assessment of the T2-W images on internal and external techniques was moderate (kappa=0.49), while the assessment of the T2-W+DW images on internal and external techniques was good (kappa $=0.62$ ).

In 26 cases who went for trachelectomy or radical hysterectomy, one case was at a different institution and information on the parametrial status was not available. Therefore, 50 parametrial regions were evaluated

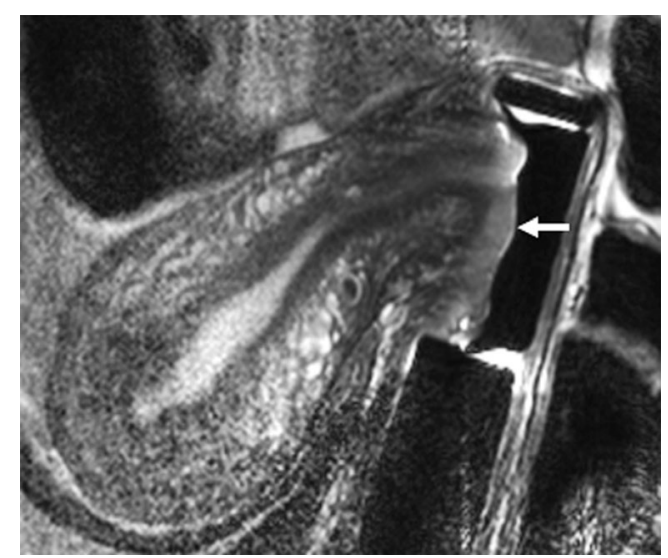

Fig. 2 True positive T2-W and T2-W+diffusion-weighted imaging (DWI) on endovaginal images missed on external array in a 25 -year-old female: Sagittal (a), axial (b), coronal (c) T2-W (FSE TR $2750 \mathrm{~ms}$, TE $80 \mathrm{~ms}$ ) and ADC map (EPI, TR $6500 \mathrm{~ms}$, TE $54 \mathrm{~ms}, \mathrm{~b}=0,100,300,500$, $800 \mathrm{~mm}^{2} / \mathrm{s}, \mathbf{d}$ ) with corresponding external array coil T2-W coronal (FSE TR $2100 \mathrm{~ms}$, TE $90 \mathrm{~ms}$, e) and ADC map (EPI TR $3230 \mathrm{~ms}$, TE $53 \mathrm{~ms}$, $\left.\mathrm{b}=0,100,300,500,800 \mathrm{~mm}^{2} / \mathrm{s}, \mathbf{f}\right)$. The intermediate signal intensity tumour in a (arrow) shows restricted diffusion in $\mathbf{d}$ (arrow). The tumour is too small for visualisation in e and f. A 17-mm tumour was confirmed on right parasagittal histopathology (g, arrow) 
Fig. 3 False positive on endovaginal $\mathrm{T} 2-\mathrm{W}$ with negative diffusion-weighted imaging (DWI) in a 41-year-old female: Sagittal (a), axial (b), coronal (c) T2-W (FSE TR $2750 \mathrm{~ms}$, TE $80 \mathrm{~ms}$ ) and ADC map (EPI, TR $6500 \mathrm{~ms}$, TE $54 \mathrm{~ms}, \mathrm{~b}=0,100$, $300,500,800 \mathrm{~mm}^{2} / \mathrm{s}$, d) with corresponding external array coil T2-W coronal (FSE TR $2100 \mathrm{~ms}$, TE $90 \mathrm{~ms}, \mathbf{e})$ and ADC map (EPI TR $3230 \mathrm{~ms}$, TE $53 \mathrm{~ms}, \mathrm{~b}=0$, $100,300,500,800 \mathrm{~mm}^{2} / \mathrm{s}$, f). The intermediate signal intensity nodule in $\mathbf{a}, \mathbf{b}$ and $\mathbf{c}$ (arrows) did not show restricted diffusion in $\mathbf{d}$ (arrow). The tumour is too small for visualisation in $\mathbf{e}$ and $\mathbf{f}$. Tumour was absent on histopathology
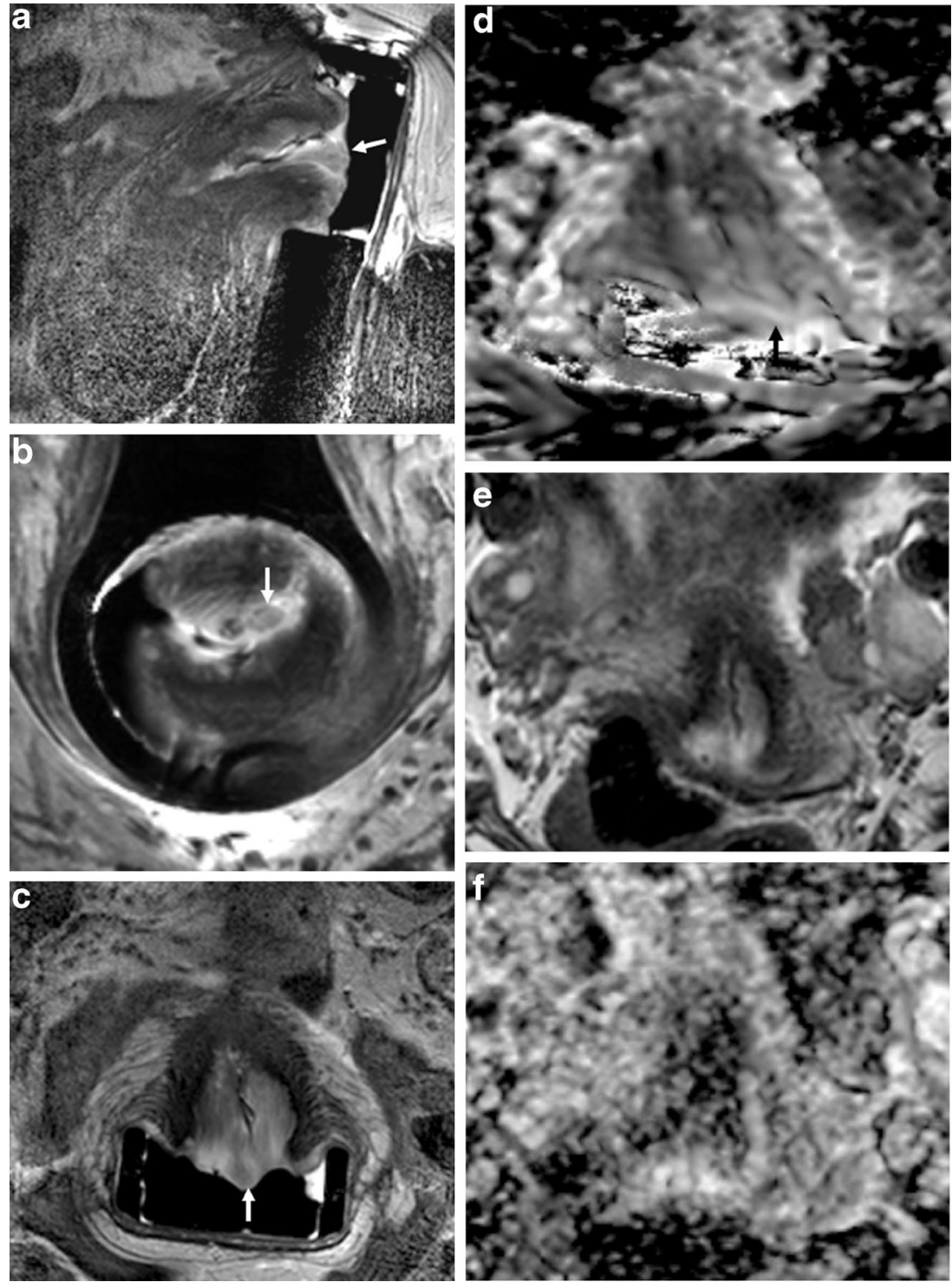

in 25 cases, assuming that right and left parametrial extension are effectively independent. Tumour within the cervix had been noted on MRI in 17 of these cases by the reporting radiologist and in 17 by the independent radiologist. Tumour within a normal-sized parametrial node was present in one case only on histology. This was missed by the reporting radiologist who marked this parametrial region as negative. The sensitivity, specificity, PPV and NPV for the independent radiologist using the endovaginal compared to the external array technique, and without and with the DWI, are given in Table 3. However, as we had only one case with parametrial extension in our cohort, no firm conclusions can be drawn about the differences in parametrial assessment with and without an endovaginal coil.

\section{Discussion}

These data clearly demonstrate a higher sensitivity of T2-W endovaginal imaging for detecting small-volume tumours compared to an external array technique, albeit with lower specificity. Importantly, however, the addition of DWI to the endovaginal imaging serves to improve specificity so that it is equivalent to that of the external array T2-W assessment, but the addition of DWI to the external array technique substantially reduces sensitivity of detection of these small lesions because the spatial resolution of DWI with external array imaging is limited by low SNR. The higher sensitivity of detection of small tumours on endovaginal T2-W imaging alone compared to external array coil imaging is to be expected because of the improved spatial resolution with the increased SNR available with a small coil in situ, and the correlation of 
Fig. 4 False negative on endovaginal $\mathrm{T} 2-\mathrm{W}$ with negative diffusion-weighted imaging (DWI) in a 27-year-old female: Sagittal (a), axial (b), coronal (c) T2-W (FSE TR $2750 \mathrm{~ms}$, TE $80 \mathrm{~ms}$ ) and ADC map (EPI, TR $6500 \mathrm{~ms}$, TE $54 \mathrm{~ms}, \mathrm{~b}=0,100$, $300,500,800 \mathrm{~mm}^{2} / \mathrm{s}$, d) with corresponding external array coil T2-W coronal (FSE TR $2100 \mathrm{~ms}$, TE $90 \mathrm{~ms}$, e) and ADC map (EPI TR $3230 \mathrm{~ms}$, TE $53 \mathrm{~ms}, \mathrm{~b}=0$, $100,300,500,800 \mathrm{~mm}^{2} / \mathrm{s}$, f).

There was no evidence of tumour on MRI with either technique, but a 6-mm microinvasive tumour (Stage Ia) was noted on histopathology (g, arrow)
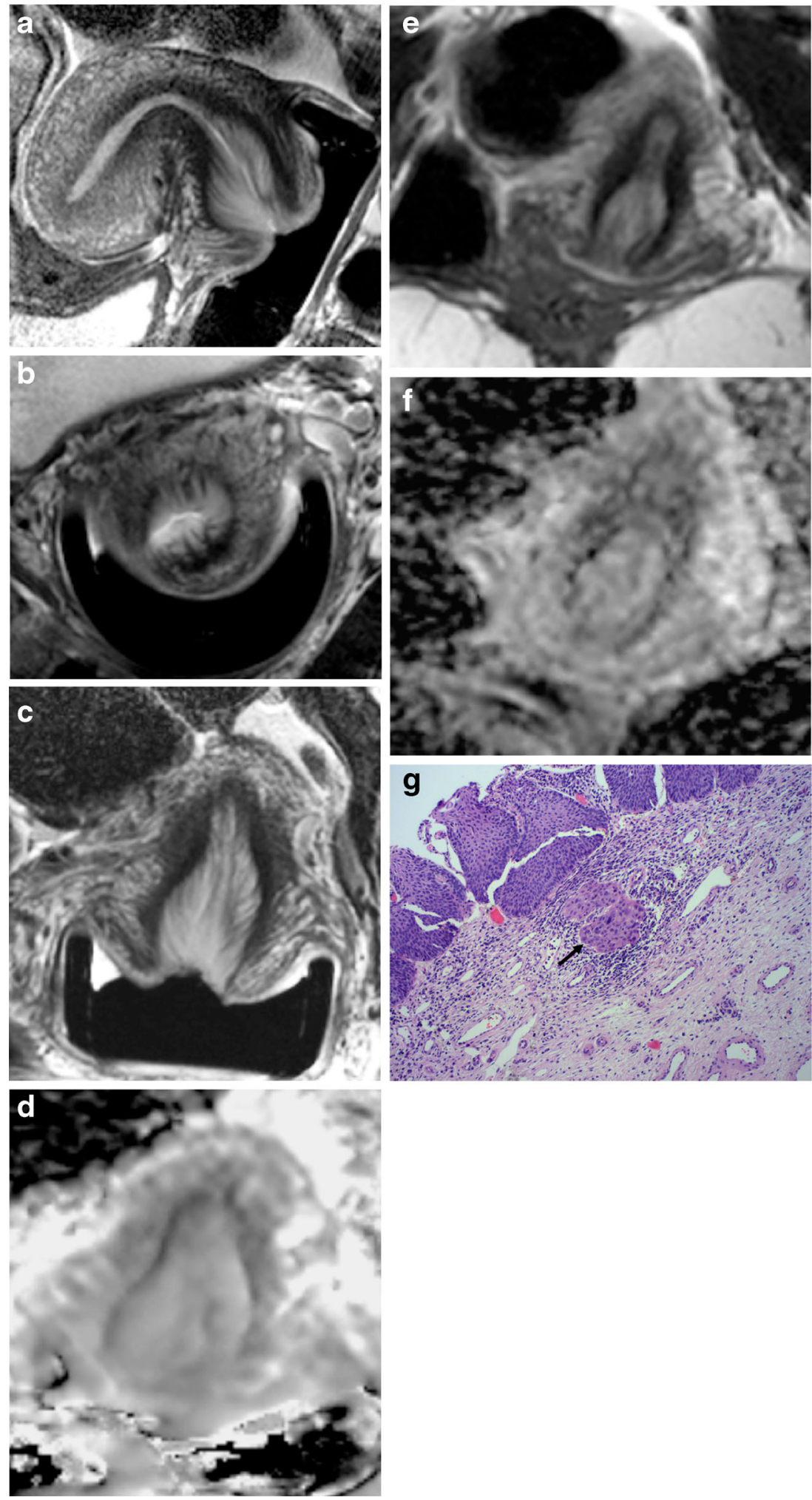

tumour size with histology has been demonstrated [20]. This has previously been shown not just in endovaginal imaging $[18,21]$ but also in prostatic imaging [22] where an endorectal technique is now standard in clinical practice.
The improved SNR afforded by an endovaginal technique also means that sequences inherently low in SNR can be more usefully deployed. In prostatic imaging the equivalent endorectal imaging has led to the routine acquisition of 
Table 2 Sensitivity, specificity, and positive (PPV) and negative (NPV) predictive values for detecting tumour by a reporting radiologist and an independent radiologist. The comparison of endovaginal vs. external

\begin{tabular}{|c|c|c|c|c|c|}
\hline \multicolumn{2}{|l|}{$\mathrm{N}=42$} & \multirow{2}{*}{$\begin{array}{l}\begin{array}{l}\text { Sensitivity } \\
\%\end{array} \\
91.3\end{array}$} & \multirow{2}{*}{$\begin{array}{l}\text { Specificity } \\
\%\end{array}$} & \multirow{2}{*}{$\begin{array}{l}\begin{array}{l}\text { PPV } \\
\%\end{array} \\
91.3\end{array}$} & \multirow{2}{*}{$\begin{array}{l}\begin{array}{l}\text { NPV } \\
\%\end{array} \\
89.5\end{array}$} \\
\hline & Reporting rad. T2W+DWI & & & & \\
\hline \multirow[t]{2}{*}{$\mathrm{T} 2-\mathrm{W}$} & Independent rad. endovaginal & 82.6 & 73.7 & 79.1 & 77.8 \\
\hline & Independent rad. external array & 73.9 & 89.5 & 89.5 & 73.9 \\
\hline \multirow[t]{2}{*}{$\mathrm{T} 2-\mathrm{W}+\mathrm{DWI}$} & Independent rad. endovaginal & 78.2 & 89.5 & 89.5 & 73.9 \\
\hline & Independent rad. external array & 66.7 & 94.7 & 94.1 & 69.2 \\
\hline
\end{tabular}

$R a d$. radiologist

multiparametric data sets which use a combination of diffusion-weighted dynamic contrast-enhanced [23, 24] and, where available, MR spectroscopic [25] techniques. Although these techniques can be acquired without endorectal imaging, this limits their sensitivity of detection to "clinically significant' prostate cancer. Lesions too small for detection by nonendorectal multiparametric imaging are deemed 'clinically insignificant'. This is not the case with cervical cancer, where even subcentimetre lesions are highly relevant and demand clinical intervention. The use of DWI in combination with endovaginal $\mathrm{T} 2-\mathrm{W}$ imaging therefore is of crucial importance in management to improve the specificity of detection of these lesions. With the external array coil alone, however, the SNR of the DWI meant that its addition to the external array T2-W imaging was detrimental in the detection of these small tumours and even provided false reassurance that a tumour was absent. Although improvements in external coil technology may well improve coil performance in future and a previous retrospective analysis of trachelectomy patients indicated a high sensitivity and specificity for the pre-operative external coil MRI [26], these improvements are unlikely to equate to the gains in SNR achievable with an internal receiver coil. The higher sensitivity and specificity of the reporting radiologist compared to the independent radiologist in our

Table 3 Sensitivity, specificity, and positive (PPV) and negative (NPV) predictive values for detecting parametrial extension by an independent radiologist. The comparison of endovaginal vs. external array techniques without and with the addition of diffusion-weighted magnetic resonance imaging (DW-MRI) to the T2-W sequences is shown

\begin{tabular}{|c|c|c|c|c|c|}
\hline \multicolumn{2}{|c|}{$\begin{array}{l}\mathrm{N}=50 \text { regions } \\
(25 \text { patients })\end{array}$} & \multirow{2}{*}{$\begin{array}{l}\begin{array}{l}\text { Sens } \\
\%\end{array} \\
100\end{array}$} & \multirow{2}{*}{$\begin{array}{l}\begin{array}{l}\text { Spec } \\
\%\end{array} \\
100\end{array}$} & \multirow{2}{*}{$\begin{array}{l}\text { PPV } \\
\%\end{array}$} & \multirow{2}{*}{$\begin{array}{l}\begin{array}{l}\text { NPV } \\
\%\end{array} \\
100\end{array}$} \\
\hline $\mathrm{T} 2-\mathrm{W}$ & Independent rad. endovaginal & & & & \\
\hline & Independent rad. external array & 100 & 95 & 33.3 & 100 \\
\hline \multirow{2}{*}{$\begin{array}{r}\text { T2-W+ } \\
\text { DWI }\end{array}$} & Independent rad. endovaginal & 100 & 100 & 100 & 100 \\
\hline & Independent rad. external array & 100 & 97.9 & 50 & 100 \\
\hline
\end{tabular}

array techniques without and with the addition of diffusion-weighted (DWI) magnetic resonance imaging to the $\mathrm{T} 2-\mathrm{W}$ sequences for the independent radiologist is shown study may in part be due to increased experience, but is also likely to have been positively influenced by the availability of clinical information at the time of reading, so that the availability of this information is recommended during reading.

A major consideration, however, is the image distortion introduced by the placement of an endovaginal probe. This is due to susceptibility in part from coil components and in part from residual air within the coil ring particularly in the region between the ring and the handle. In addition, residual air in the vagina introduced during coil placement further exacerbates the problem. The latter was minimised by sucking out residual air using a Ryles tube prior to imaging. This collapses the vaginal wall around the coil handle and minimises residual vaginal air, although it is not fool-proof and small pockets of air remain, particularly in the vaginal fornices. The coil construction uses MR compatible components with virtually no artefact on prior testing, so it is assumed that their contribution to susceptibility-induced distortions is minimal. Improved coil design and construction to minimise air around components should further reduce these problems.

Another approach to improving geometric distortions on the DW images [27] is to replace the echo-planar based sequences with a turbo spin-echo (TSE). Previous attempts at such an approach have resulted in successful implementation of turbo spin-echo DWI for head and neck [28] and endometrial [29] cancers. The resulting images are more robust and suffer less from distortion, but incur a time penalty. Typically an increase of around $50 \%$ in imaging time is expected. There is also an increased tendency to motion artefact and as these images are not single-shot like their echo-planar based counterpart, there is increased image blurring [30]. Optimisation of the DW technique for endovaginal MRI is therefore warranted; evaluation of the advantages and limitations of an EPI versus TSE-based sequences is required but was beyond the scope of the study, not least because of the length of a detailed endovaginal examination followed by a detailed external array examination. The number of $b$-values to use is also debated [31], but the choice of five b-values in this instance was based 
on the ability to calculate ADC more accurately, even though quantitative assessments did not form part of the current work.

The scan schedule in this small group of patients was intensive and took $\sim 60 \mathrm{~min}$ but fortunately would not be required in standard clinical care. It is our normal practise to do a detailed evaluation of the cervix using the endovaginal coil over $25 \mathrm{~min}$ and to then remove it and assess the pelvic and abdominal lymph node chains with an external array, which takes another $20 \mathrm{~min}$. Although longer than a standard external array pelvic MRI of $30 \mathrm{~min}$, this has the added value of detailed data prior to planning a fertility-sparing surgical procedure such as a trachelectomy in these young women.

Appropriate referral for an endovaginal MRI is largely based on an approximate clinical or histological assessment of tumour volume. Larger tumours, particularly stage $2 \mathrm{~b}$ and above, are adequately imaged using an external array coil technique $[32,33]$. Tumours benefiting from this procedure are predominantly those clinically staged as Ia 2 or Ib1, where the tumour may or may not be just palpable or the diagnostic or therapeutic cone biopsy or LLETZ indicates that there is incomplete excision of tumour or tumour extending to margins of the specimen. In these cases, endovaginal MRI is invaluable in depicting the presence of residual tumour and defining its extent in relation to the endocervical canal pre-operatively. As referrals to our study were largely based on these criteria, the tumour median volume in our cohort was $1.75 \mathrm{~cm}^{3}$. The increased detectability of these small tumours with endovaginal MRI is directly related to the improved spatial resolution possible because of the substantial improvement in SNR afforded by the internal coil. Although the numbers of patients are small, the sensitivity and specificity of our endovaginal reads by the reading radiologist are identical to those of previous cohorts studied with this technique [20].

\section{Conclusion}

Sensitivity for detecting tumour within the cervix was higher with an endovaginal coil technique than with a conventional external array coil, although specificity was lower. The addition of DWI to the endovaginal T2-W imaging gave a small reduction in sensitivity, but with a substantial improvement in specificity, making this the preferred diagnostic option. Although less sensitive, specificity for tumour detection was high with the external array coil using T2-W imaging; the addition of DWI in this case did not improve specificity, but markedly reduced sensitivity. Therefore DWI is only useful in early stage cervical cancer as an adjunct to T2-W imaging if an endovaginal technique is used. Endovaginal coil MRI would be recommended for assessment of clinical stage $1 \mathrm{a}$ and $1 \mathrm{~b} 1$ cervical cancer.
Acknowledgements The authors thank Dr D.J. Gilderdale and Mr C. Cummings for design and manufacture of the endovaginal coil. This work was supported by Medical Research Council Grant No. G0802470.

The scientific guarantor of this publication is Nandita deSouza. The authors of this manuscript declare no relationships with companies whose products or services may be related to the subject matter of this article. This study has received funding from the Medical Research Council Grant No. G0802470. The Imaging Centre is supported by CRUK \& EPSRC in association with MRC and Dept of Health C1060/A10334 and NHS funding to the NIHR Clinical Research Facility in Imaging. No complex statistical methods were necessary for this paper. Institutional Review Board approval was obtained. Written informed consent was obtained from all patients in this study. Methodology: Prospective, diagnostic study performed at one institution.

Open Access This article is distributed under the terms of the Creative Commons Attribution-NonCommercial 4.0 International License (http://creativecommons.org/licenses/by-nc/4.0/), which permits any noncommercial use, distribution, and reproduction in any medium, provided you give appropriate credit to the original author(s) and the source, provide a link to the Creative Commons license, and indicate if changes were made.

\section{References}

1. Abu-Rustum NR, Neubauer N, Sonoda Y, Park KJ, Gemignani M, Alektiar KM, Tew W, Leitao MM, Chi DS, Barakat RR (2008) Surgical and pathologic outcomes of fertility-sparing radical abdominal trachelectomy for FIGO stage IB1 cervical cancer. Gynecol Oncol 111:261-264

2. Gien LT, Covens A (2010) Fertility-sparing options for early stage cervical cancer. Gynecol Oncol 117:350-357

3. Shepherd JH, Spencer C, Herod J, Ind TE (2006) Radical vaginal trachelectomy as a fertility-sparing procedure in women with earlystage cervical cancer-cumulative pregnancy rate in a series of 123 women. BJOG 113:719-724

4. Bipat S, Van Den Berg RA, Van Der Velden J, Stoker J, Spijkerboer AM (2011) The role of magnetic resonance imaging in determining the proximal extension of early stage cervical cancer to the internal os. Eur J Radiol 78:60-64

5. Sheu MH, Chang CY, Wang JH, Yen MS (2001) Preoperative staging of cervical carcinoma with MR imaging: a reappraisal of diagnostic accuracy and pitfalls. Eur Radiol 11:1828-1833

6. Vargas HA, Akin O, Zheng J, Moskowitz C, Soslow R, AbuRustum N, Barakat RR, Hricak H (2011) The value of MR imaging when the site of uterine cancer origin is uncertain. Radiology 258: 785-792

7. Peppercorn PD, Jeyarajah AR, Woolas R, Shepherd JH, Oram DH, Jacobs IJ, Armstrong P, Lowe D, Reznek RH (1999) Role of MR imaging in the selection of patients with early cervical carcinoma for fertility-preserving surgery: initial experience. Radiology 212: 395-399

8. Hricak H, Gatsonis C, Coakley FV, Snyder B, Reinhold C, Schwartz LH, Woodward PJ, Pannu HK, Amendola M, Mitchell DG (2007) Early invasive cervical cancer: CT and MR imaging in preoperative evaluation - ACRIN/GOG comparative study of diagnostic performance and interobserver variability. Radiology 245 : 491-498

9. Sahdev A, Sohaib SA, Wenaden AE, Shepherd JH, Reznek RH (2007) The performance of magnetic resonance imaging in early cervical carcinoma: a long-term experience. Int J Gynecol Cancer 17:629-636 
10. deSouza NM, Dina R, Mcindoe GA, Soutter WP (2006) Cervical cancer: value of an endovaginal coil magnetic resonance imaging technique in detecting small volume disease and assessing parametrial extension. Gynecol Oncol 102:80-85

11. deSouza NM, Scoones D, Krausz T, Gilderdale DJ, Soutter WP (1996) High-resolution MR imaging of stage I cervical neoplasia with a dedicated transvaginal coil: MR features and correlation of imaging and pathologic findings. AJR Am J Roentgenol 166:553559

12. deSouza NM, Whittle M, Williams AD, Sohail M, Krausz T, Gilderdale DJ, Mcindoe GA, Soutter WP (2000) Magnetic resonance imaging of the primary site in stage I cervical carcinoma: a comparison of endovaginal coil with external phased array coil techniques at 0.5T. J Magn Reson Imaging 12:1020-1026

13. Soutter WP, Hanoch J, D'arcy T, Dina R, Mcindoe GA, deSouza NM (2004) Pretreatment tumour volume measurement on highresolution magnetic resonance imaging as a predictor of survival in cervical cancer. BJOG 111:741-747

14. Gilderdale DJ, deSouza NM, Coutts GA, Chui MK, Larkman DJ, Williams AD, Young IR (1999) Design and use of internal receiver coils for magnetic resonance imaging. Br J Radiol 72:1141-1151

15. Downey K, Jafar M, Attygalle AD, Hazell S, Morgan VA, Giles SL, Schmidt MA, Ind TE, Shepherd JH, deSouza NM (2013) Influencing surgical management in patients with carcinoma of the cervix using a T2- and ZOOM-diffusion-weighted endovaginal MRI technique. Br J Cancer 109:615-622

16. Naganawa S, Sato C, Kumada H, Ishigaki T, Miura S, Takizawa O (2005) Apparent diffusion coefficient in cervical cancer of the uterus: comparison with the normal uterine cervix. Eur Radiol 15:7178

17. Payne GS, Schmidt M, Morgan VA, Giles S, Bridges J, Ind T, deSouza NM (2010) Evaluation of magnetic resonance diffusion and spectroscopy measurements as predictive biomarkers in stage 1 cervical cancer. Gynecol Oncol 116:246-252

18. Charles-Edwards EM, Messiou C, Morgan VA, De Silva SS, Mcwhinney NA, Katesmark M, Attygalle AD, deSouza NM (2008) Diffusion-weighted imaging in cervical cancer with an endovaginal technique: potential value for improving tumor detection in stage Ia and Ib1 disease. Radiology 249:541-550

19. Wheeler-Kingshott CA, Parker GJ, Symms MR et al (2002) ADC mapping of the human optic nerve: increased resolution, coverage, and reliability with CSF-suppressed ZOOM-EPI. Magn Reson Med 47:24-31

20. Downey K, Shepherd JH, Attygalle AD, Hazell S, Morgan VA, Giles SL, Ind TE, deSouza NM (2014) Preoperative imaging in patients undergoing trachelectomy for cervical cancer: validation of a combined T2- and diffusion-weighted endovaginal MRI technique at 3.0 T. Gynecol Oncol 133:326-332

21. Charles-Edwards E, Morgan V, Attygalle AD, Giles SL, Ind TE, Davis M, Shepherd J, Mcwhinney N, deSouza NM (2011) Endovaginal magnetic resonance imaging of stage $1 \mathrm{~A} / 1 \mathrm{~B}$ cervical cancer with A T2- and diffusion-weighted magnetic resonance technique: effect of lesion size and previous cone biopsy on tumor detectability. Gynecol Oncol 120:368-373

22. Turkbey B, Merino MJ, Gallardo EC, Shah V, Aras O, Bernardo M, Mena E, Daar D, Rastinehad AR, Linehan WM, Wood BJ, Pinto
PA, Choyke PL (2014) Comparison of endorectal coil and nonendorectal coil T2W and diffusion-weighted MRI at 3 Tesla for localising prostate cancer: correlation with whole-mount histopathology. J Magn Reson Imaging 39:1443-1448

23. Korn N, Kurhanewicz J, Banerjee S, Starobinets O, Saritas E, Noworolski S (2015) Reduced-FOV excitation decreases susceptibility artifact in diffusion-weighted MRI with endorectal coil for prostate cancer detection. Magn Reson Imaging 33:56-62

24. Nowak J, Malzahn U, Baur AD, Reichelt U, Franiel T, Hamm B, Durmus T (2014) The value of ADC, T2 signal intensity, and a combination of both parameters to assess Gleason score and primary Gleason grades in patients with known prostate cancer. Acta Radiol

25. Riches SF, Payne GS, Morgan VA, Sandhu S, Fisher C, Germuska M, Collins DJ, Thompson A, deSouza NM (2009) MRI in the detection of prostate cancer: combined apparent diffusion coefficient, metabolite ratio, and vascular parameters. AJR Am J Roentgenol 193:1583-1591

26. Lakhman Y, Akin O, Park KJ, Sarasohn DM, Zheng J, Goldman DA, Sohn MJ, Moskowitz CS, Sonoda Y, Hricak H, Abu-Rustum NR (2013) Stage IB1 cervical cancer: role of preoperative MR imaging in selection of patients for fertility-sparing radical trachelectomy. Radiology 269:149-158

27. Malkyarenko DI, Chenevert TL (2014) Practical estimate of gradient nonlinearity for implementation of apparent diffusioncoefficient bias correction. J Magn Reson Imaging 40:1487-1495

28. Sakamoto J, Imaizumi A, Sasaki Y, Kamio T, Wakoh M, OtonariYamamoto M, Sano T (2014) Comparison of accuracy of intravoxel incoherent motion and apparent diffusioncoefficient techniques for predicting malignancy of head and neck tumors using half-Fourier single-shot turbo spin-echo diffusion-weighted imaging. Magn Reson Imaging 32:860-866

29. Hori M, Kim T, Onishi H, Imaoka I, Kagawa Y, Murakami T, Nakamoto A, Ueguchi T, Tatsumi M, Enomoto T, Kimura T, Tomiyama N (2013) Endometrial cancer: preoperative staging using three-dimensional T2-weighted turbo spin-echo and diffusion-weighted MR imaging at 3.0 T: a prospective comparative study. Eur Radiol 23:2296-2305

30. Lee CY, Li Z, Pipe JG, Debbins JP (2013) Turboprop+: enhanced Turboprop diffusion-weighted imaging with a new phase correction. Magn Reson Med 70:497-503

31. Hoogendam JP, Klerkx WM, De Kort GA, Bipat S, Zweemer RP, Sie-Go DM, Verheijen RH, Mali WP, Veldhuis WB (2010) The influence of the b-value combination on apparent diffusion coefficient based differentiation between malignant and benign tissue in cervical cancer. J Magn Reson Imaging 32:376-382

32. Hori M, Kim T, Murakami T, Imaoka I, Onishi H, Tomoda K, Tsutsui T, Enomoto T, Kimura T, Nakamura H (2009) Uterine cervical carcinoma: preoperative staging with 3.0-T MR imagingcomparison with 1.5-T MR imaging. Radiology 251:96-104

33. Mitchell DG, Snyder B, Coakley F, Reinhold C, Thomas G, Amendola M, Schwartz LH, Woodward P, Pannu H, Hricak H (2006) Early invasive cervical cancer: tumor delineation by magnetic resonance imaging, computed tomography, and clinical examination, verified by pathologic results, in the ACRIN 6651/GOG 183 Intergroup Study. J Clin Oncol 24:5687-5694 